\title{
An experimental investigation on elliptical instability of a strongly asymmetric vortex pair in a stable density stratification
}

\author{
B. Cariteau and J.-B. Flór \\ Laboratoire des Ecoulements Géophysiques et Industriels, PB53 38041 Grenoble Cedex 9, France
}

Received: 1 August 2005 - Revised: 29 June 2006 - Accepted: 7 November 2006 - Published: 20 November 2006

\begin{abstract}
We investigate the elliptical instability of a strongly asymmetric vortex pair in a stratified fluid, generated by the acceleration and deceleration of the rotation of a single flap. The dominant parameter is the Froude number, $F r=U /(N R)$, based on the maximum azimuthal velocity, $U$, and corresponding radius, $R$, of the strongest vortex, i.e. the principal vortex, and buoyancy frequency $N$. For $F r>1$, both vortices are elliptically unstable while the instability is suppressed for $F r<1$. In an asymmetric vortex pair, the principal vortex is less - and the secondary vortex more - elliptical than the vortices in an equivalent symmetric dipolar vortex. The far more unstable secondary vortex interacts with the principal vortex and increases the strain on the latter, thus increasing its ellipticity and its instability growth rate. The nonlinear interactions render the elliptical instability more relevant. An asymmetric dipole can be more unstable than an equivalent symmetric dipole. Further, the wavelength of the instability is shown to be a function of the Froude number for strong stratifications corresponding to small Froude numbers, whereas it remains constant in the limit of a homogenous fluid.
\end{abstract}

\section{Introduction}

The stability of monopolar vortices in homogeneous and stratified fluids is of relevance to transport and mixing processes in geophysical and turbulent flows. In particular dipolar and monopolar vortices appear abundantly in the Earth oceans and atmosphere. These structures are self propelling, or propagate under the influence of the Coriolis force, and may transport heat as well as chemical and biological compounds over large distances. Stability prolongs their longevity, whereas instability favors the local mixing

Correspondence to: J.-B. Flór

(flor@hmg.inpg.fr) and the energy transfer to small scales. In this paper, we focus on the influence of a stable vertical density-stratification on the elliptical instability of an asymmetric dipole.

Elliptical instability is an essentially three dimensional instability which arises due to the parametric resonance between the oscillation of inertial waves in the horizontal flow and the ambient strain field (see the review of Kerswell, 2002). For open flows such as a moving dipolar vortex, this instability has been first demonstrated experimentally by Leweke and Williamson (1998). In a stratified fluid, these oscillations (inertia-gravity waves) are only permitted in the frequency range between the buoyancy frequency and rotation frequency set by the rotation of the vortex. Strong stratification "closes" this frequency gap, within which resonance may occur and thereby suppresses the elliptical instability (see Miyazaki and Fukumoto, 1992; Kerswell, 1994, 2002).

The effect of stratification on the growth rate of elliptical instability has been investigated numerically by Potylitsin and Peltier (1998) for the case of monopolar vortices (Kelvin Helmholtz and Kida vortex) in the presence of a weak stratification, and for dipolar vortices in the presence of a strong stratification by Billant and Chomaz (2000b). Billant and Chomaz showed that the wave length of the elliptical instability increases, while the growth rate decreases with $1 / F r$, for Froude numbers decreasing to 0.22 . This Froude number is defined as $F r=U_{0} /\left(N R_{D}\right)$ and is based on the translation velocity, $U_{0}$, and radius $R_{D}$ of the Lamb-Chaplygin-dipole. Using the velocity on the dipole axis which is $\left(2.49 U_{0}\right)$ and half the radius of the dipole, this Froude number is approximately five times larger than the monopole Froude number, explaining the lower value of 0.22 in their experiments. The equivalent criterion for a monopole is $F r=1$ derived by Miyazaki and Fukumoto (1992) for a uniform rotation. For Froude-number values, $F r \leq 0.22$ (and $F r \approx 1$ for a monopolar vortex), elliptical instability is suppressed in agreement with Miyazaki and Fukumoto (1992), and the zigzag instability dominates. In the sequel of this paper we

Published by Copernicus GmbH on behalf of the European Geosciences Union and the American Geophysical Union. 


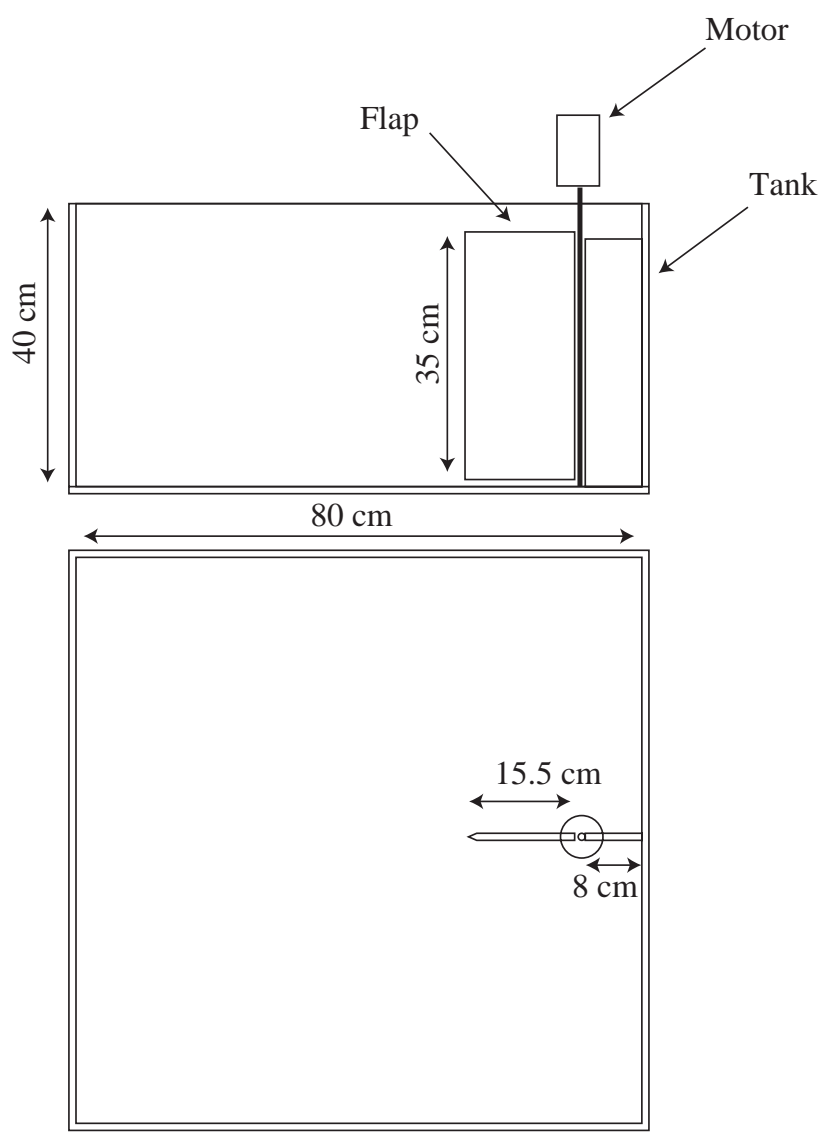

Fig. 1. Sketch of the side-view and top-view of the experimental setup and stratification. The flap was either rotated by hand or by a computer controlled DC-motor.

will employ the monopole Froude number mentioned above, based on maximum azimuthal velocity $U$ and corresponding radius R. In a theoretical study Kerswell (1992) considers the elliptical instability of stratified and hydromagnetic waves for a solid body rotation. Among the different modes investigated, the growth rate of the core mode, which is of interest here, decreases with increased stratification.

Our research is at variance with the numerical results of Billant and Chomaz (2000) in that we consider strongly asymmetric vortices in which the partner vortex can be considered as secondary. This secondary vortex becomes unstable in accompaniment of the principal vortex and, while being entrained, significantly increases the strain on the principal vortex. We investigate the evolution of the principal vortex in this rather perturbed state. After presenting the experimental setup in the next section, the detailed flow properties are discussed in Sect. 3. The elliptical instability of the principal vortex is described in Sect. 4 , followed by the conclusions in Sect. 5.

\section{Experimental setup}

Two experimental setups are used. A first set of experiments is conducted at the DAMTP laboratory in Cambridge in a tank of dimensions $40 \times 40 \times 40 \mathrm{~cm}^{3}$. In a next set, a tank of dimensions $80 \times 80 \times 40 \mathrm{~cm}^{3}$ is used (see Fig. 1). In both cases, the tank is filled with a stable density-stratified fluid, with tap-water as working fluid, the stratification obtained by a linear variation in salt concentration using the doublebucket method (Oster, 1965). The total fluid-height is 30 to $35 \mathrm{~cm}$. To inhibit axial flow due to Ekman pumping near top and bottom, a relatively dense salt-saturated layer is added at the bottom, and a layer of fresh water at the free surface. This reduces the working depth to 25 to $30 \mathrm{~cm}$. The buoyancy frequency, defined by $N^{2}=-g / \bar{\rho} d \rho / d z$ with density $\rho(z)$ and mean density $\bar{\rho}$, is measured by a linear regression of $10 \mathrm{den}$ sity samples regularly spaced over the total working depth.

The vortex structure is generated far from the boundaries and over the entire tank depth by a flap of $15.5 \mathrm{~cm}$ width and $35 \mathrm{~cm}$ height. The flap axis is placed vertically, with a precision up to approximatively 0.05 radians corresponding to $0.3 \mathrm{~mm}$ over the flap height. The axis is $8 \mathrm{~cm}$ from the nearest wall (see Fig. 1). In both experiments the same flap is used. In the first set of experiments the flap is shortly moved by hand such that it produced a very a-symmetric dipole and initial conditions are measured just after vortex generation by means of particle-tracking measurements using the software package DigImage (Dalziel, 1993). These experimental results are only used for the data in Fig. 10.

In the second set of experiments, the flap is driven by a computer controlled DC-motor, and programmed to produce a constant acceleration followed by a $1 / t$ deceleration up to a constant very low velocity. A principal vortex is produced during acceleration (starting vortex) and a smaller secondary vortex (stopping vortex) during deceleration. Depending on the forcing, the stopping vortex can be as intense as the starting vortex and results in a nearly symmetric vortex pair (see e.g. Thomas and Auerbach, 1994; Afanasyev, 2002). The decrease of the flap velocity is smooth in order to minimize the intensity of the stopping vortex and to increase the separation distance from the starting vortex. The duration of the acceleration stage is 0.5 or $2 \mathrm{~s}$ and the maximum angular velocity is varied from 0.2 to $0.27 \mathrm{rad} / \mathrm{s}$. The final angular velocity of the flap is $0.08 \mathrm{rad} / \mathrm{s}$ and the total duration of the motion is 50 to $60 \mathrm{~s}$.

In order to measure the oscillations of the principal vortex core, dye is generated along the long edge of the flap, either with the solder wire technique (see Honyi et al., 1980) or with a cotton wire containing fluorescent dye. During the vortex formation, this thin sheet of dye is continuously entrained within the principal vortex, visualizing the core as well as the vortex motion at larger radii. To simultaneously measure the horizontal velocity field, and in particular the vortex maximum azimuthal velocity and corresponding radius, the motion of particles seeded at mid-depth, illuminated 

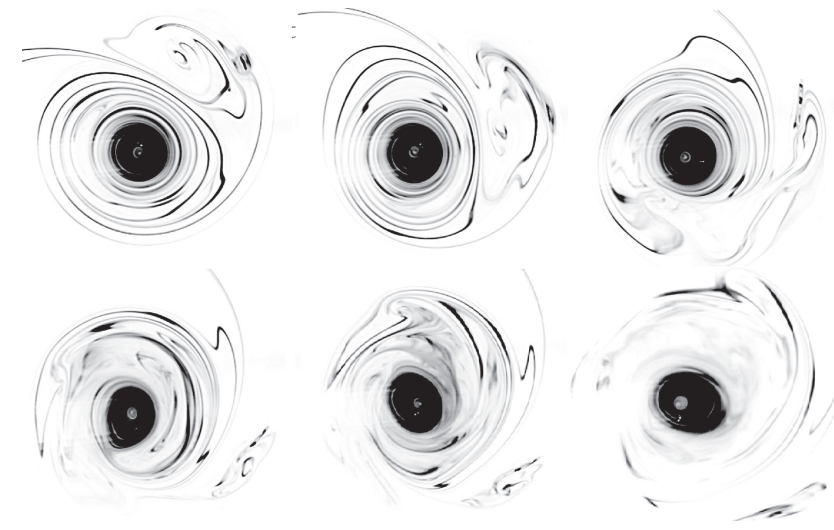

Fig. 2. Sequence of top-view dye-visualizations of the asymmetric dipolar flow for Froude number approximately 4. The flow was illuminated at mid-height with a laser sheet. The images, from left to right and top to bottom, are taken at times 30, 35, 41, 46, 49 and $51 \mathrm{~s}$, respectively.

by a $5 \mathrm{~mm}$ to $10 \mathrm{~mm}$-thick sheet of light (laser, or projector), is recorded from the top. Either a second camera is placed above the tank, or one single camera recorded top view and side view in one image via a mirror that was placed under an angle of 45 degrees with the side-wall of the tank. The particle sizes are either of about $60 \mu \mathrm{m}$ or $200 \mu \mathrm{m}$ in diameter. The images are recorded with $30 \mathrm{~Hz}, 768 \times 484 \mathrm{pxl}, 8$ bit and a $25 \mathrm{~Hz} 768 \times 572$ pxl, 8 bit CCD camera. In the second set of experiments, the horizontal velocity field is obtained using the particle image velocimetry (C.I.V.) technique developed by Fincham and Spedding (1997) and Fincham and Delerce (2000). In order to follow the evolution in the density field, side-view shadow-graph visualizations are recorded, whereas in some cases the "synthetic Schlieren" method (see e.g. Dalziel et al., 2000) is employed. The experimental data of both experimental sets is listed in Table 1 .

\section{Observations}

The top-view dye visualizations in Fig. 2 show, that once the flap has ceased to move, a circular dye-line encloses the principal and secondary vortex of the asymmetric dipolar structure. The secondary vortex is elliptical and, in the presence of the strain field of the principal vortex, becomes elliptically unstable (Fig. $2 t=35 \mathrm{~s}, 41 \mathrm{~s}$ ). The amplitude of the instability grows such that the secondary vortex deviates in horizontal direction. Meanwhile the secondary vortex slightly approaches the principal vortex, while locally mixing the dye pattern, showing a three dimensional motion. The core of the principal vortex is initially circular, and becomes elliptical in particular between 41 and $46 \mathrm{~s}$.

The synthetic Schlieren pictures in Fig. 3 reveal the vertical evolution of the instability of the secondary vortex. The core of the secondary vortex is marked by the black line,
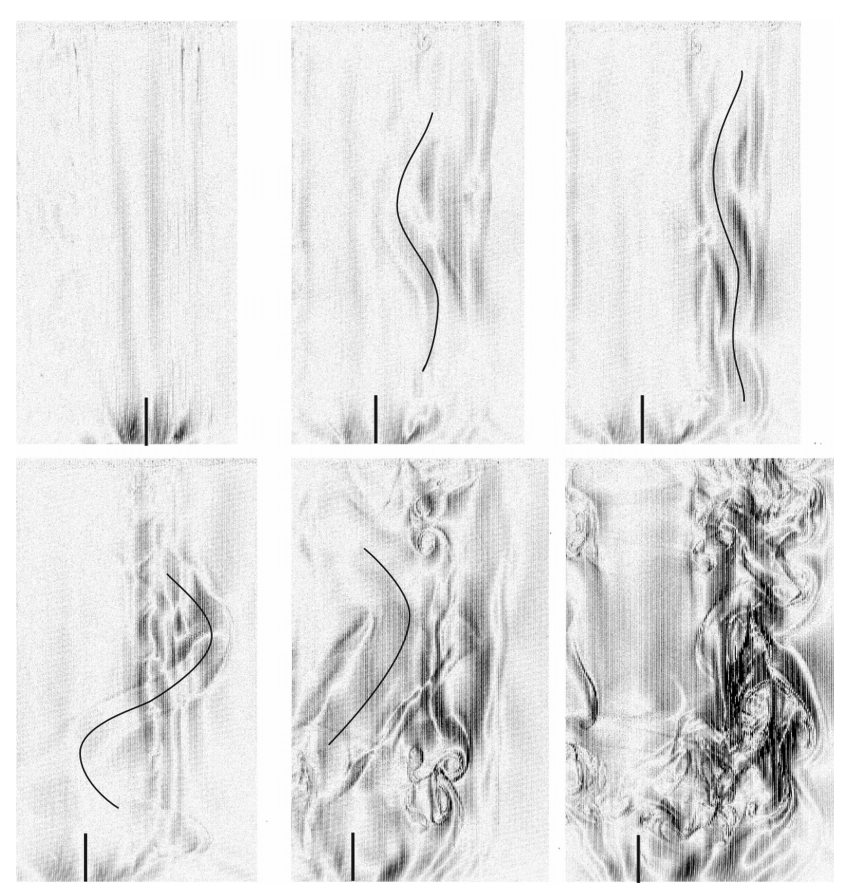

Fig. 3. Sequence of (side-view) synthetic Schlieren visualizations for a Froude number close to 4 . The vertical line at the bottom of each image points at the principal vortex, whereas the curve in the second image indicates the center of the secondary vortex. Corresponding times are 25, 34, 36, 41, 51 and $57 \mathrm{~s}$ from left to right and top to bottom. The height of the images is $22 \mathrm{~cm}$.

whereas the presence of the principal vortex can be discerned from the grey vertical lines in the background. The sequence clearly shows the secondary vortex wrapping around the principal vortex in a similar way as elliptical unstable vortices in a homogeneous rotating fluid (see Afanasyev, 2002). The wavelength is of the order 4 to 5 times the vortex radius (i.e. $\approx 9 \mathrm{~cm}$ ). Since the principal vortex was not perturbed, and the wave length is much shorter than the vortex separation distance of about $10 \mathrm{~cm}$, the Crow instability can be excluded. The secondary vortex approaches the principal vortex to such an extent that instabilities occur locally, leading to the mushroom shaped structures along the principal vortex (Fig. 3 at $t=51 \mathrm{~s}$ ). Eventually, the secondary vortex breaks down in the vicinity of the principal vortex, creating an ambient region of turbulence $(t=57 \mathrm{~s})$.

The principal vortex increases in ellipticity once the secondary vortex becomes unstable indicating that the applied strain increases until the principal vortex becomes unstable. Figure $4 \mathrm{a}$ shows a dye visualization of the principal vortex core before and after the onset of the oscillations. The corresponding spatiotemporal evolution of the core line (see Fig. 4b) shows initially inertial waves (Kelvin waves) for $10 \mathrm{~s}<t<30 \mathrm{~s}$ and the growing instability for $t>30 \mathrm{~s}$. A vertical cross section of the principal vortex structure in Fig. 5 reveals the typical radial structure for elliptical instability, 
Table 1. Table of the initial buoyancy frequency $N$, measured velocity $U$, radius $R$ and wavelength $\lambda$. The circulation is estimated from the radius and maximum velocity using the Oseen vortex model. The last five experiments correspond to the experiments conducted in the small tank.

\begin{tabular}{ccccccccc}
\hline$N(\mathrm{rad} / \mathrm{s})$ & $U(\mathrm{~cm} / \mathrm{s})$ & $R(\mathrm{~cm})$ & $\Gamma\left(\mathrm{cm}^{2} / \mathrm{s}\right)$ & $\lambda(\mathrm{cm})$ & $F r=U /(N R)$ & $\lambda / R$ & $\operatorname{Re}=U R / \nu$ & $\operatorname{Re}_{\Gamma}=\Gamma / \nu$ \\
\hline 0.63 & 3.2 & 2.4 & 67 & 5.7 & 2.0 & 2.4 & 770 & 6700 \\
0.63 & 4.5 & 2.2 & 87 & 4.9 & 3.2 & 2.2 & 1000 & 8700 \\
0.54 & 6.2 & 2.2 & 120 & 5.0 & 5.2 & 2.3 & 1360 & 12000 \\
0.63 & 5.7 & 1.8 & 90 & 4.9 & 5.0 & 2.5 & 1000 & 9000 \\
0.54 & 5.4 & 2.0 & 95 & 5.2 & 5.0 & 2.6 & 1100 & 9500 \\
1.21 & 2.5 & 1.5 & 76 & 4.5 & 1.3 & 3.0 & 380 & 7600 \\
1.21 & 3.8 & 2.1 & - & 4.5 & 1.5 & 2.17 & 800 & - \\
1.0 & 3.8 & 2.7 & - & 8.8 & 1.1 & 3.3 & 1030 & - \\
0.58 & 2.2 & 2.4 & - & 5.5 & 1.6 & 2.4 & 530 & - \\
0.58 & 2.8 & 2.6 & - & 4.8 & 1.9 & 1.9 & 730 & - \\
\hline
\end{tabular}

characterized by an unperturbed stream tube with a diameter of half the wavelength, also observed by Leweke and Williamson (1998). The present Froude number $>0.5$ and the short wavelength, exclude the zig-zag instability. The structure and relative size of the unstable mode in Fig. 5 are reminiscent of the elliptical instability observed by Leweke and Williamson (1998). In addition the suppression of the instability for strong stratification (see Miyazaki and Fukumoto, 1992) (see Fig. 2) suggest an elliptical instability.

During the flow evolution, the Froude number decreases due to viscous dissipation, and the wavelength of the elliptical instability was observed to increase accordingly. As a consequence, the instability of the principal vortex never resulted in a complete vortex breakdown but saturated. For small Froude numbers $(F r<1)$ the zigzag instability (Billant and Chomaz, 2000a) was observed eventually.

\section{Flow properties of the principal vortex}

\subsection{The vortex velocity profile}

In order to characterize the vortex generated by the flapmotion, the horizontal velocity field was measured using the CIV technique mentioned above. Figure 6 shows instantaneous cross-sectional velocity-profiles, of three different experiments taken at $12 \mathrm{~s}$ after forcing. Each profile is obtained from an azimuthal average. These experimental profiles are compared to a theoretical model, which is an analytical solution of the two-dimensional diffusion equation (Kloosterziel, 1990; Flór and Eames, 2002), given by:

$v(r)=W i_{m, n}\left(r^{2} / 2\right) e^{-r^{2} / 4}$,

with $W i_{m, n}$ the Whittaker function where $n=0.5$ and $m=(\beta-1) / 2$. The parameter $\beta$ controls the decrease of the azimuthal velocity with $r$. For $\beta=1$ the velocity field decays as $1 / r$, while for $\beta=3$ the equation represents a Gaussian velocity-profile. The parameters $\beta, U$, and $R$ are deduced from a fit of Eq. (1) with the experimental data. In Fig. 6 the continuous curves represent the fit and the dashed curves the corresponding theoretical vorticity-profiles. In the experiments here reported, $\beta$ is close to 0.7 for the three different experiments. With time, the velocity and radius vary due to viscous diffusion of vorticity, but the velocity profile remains self similar and the evolution is close to that of a twodimensional vortex with a Gaussian vorticity-distribution.

After vortex formation at about $10 \mathrm{~s}$, typically the maximum azimuthal velocity is $\approx 5 \mathrm{~cm} / \mathrm{s}$ at radius $R \approx 2 \mathrm{~cm}$ yielding a Reynolds number, $R e=U R / \nu=1000$. This Reynolds number remains about constant up to the onset of the instability, and decays slightly during the subsequent flow evolution under consideration to values of order 800 . Viscous effects on the scale of the vortex are further neglected.

\subsection{The initial strain induced by the secondary vortex}

A typical example of an asymmetric dipole is shown in the stream line plot in Fig. 7. The principal vortex is weakly elliptical whereas the weaker secondary vortex is strongly elliptical, and thus both vortices are susceptible to elliptic instability.

To provide an estimation of the growth rate based on existing theoretical results we use the theoretical results by Kerswell (1992 and 2002) and le Dizès and Laporte (2002). Kerswell (1992) uses a basic state of solid body rotation in a rotating stratified environment. le Dizès and Laporte (2002) use a general pair of Gaussian vortices in a homogeneous fluid.

For a period of $20 \mathrm{~s}$ during which the separation distance is approximatively constant, the experimental dipole trajectory can be modeled by a pair of point-vortices. The theoretical angular velocity is

$\Omega_{p}=\frac{\Gamma_{1}+\Gamma_{2}}{2 \pi b_{e q}^{2}}$, 

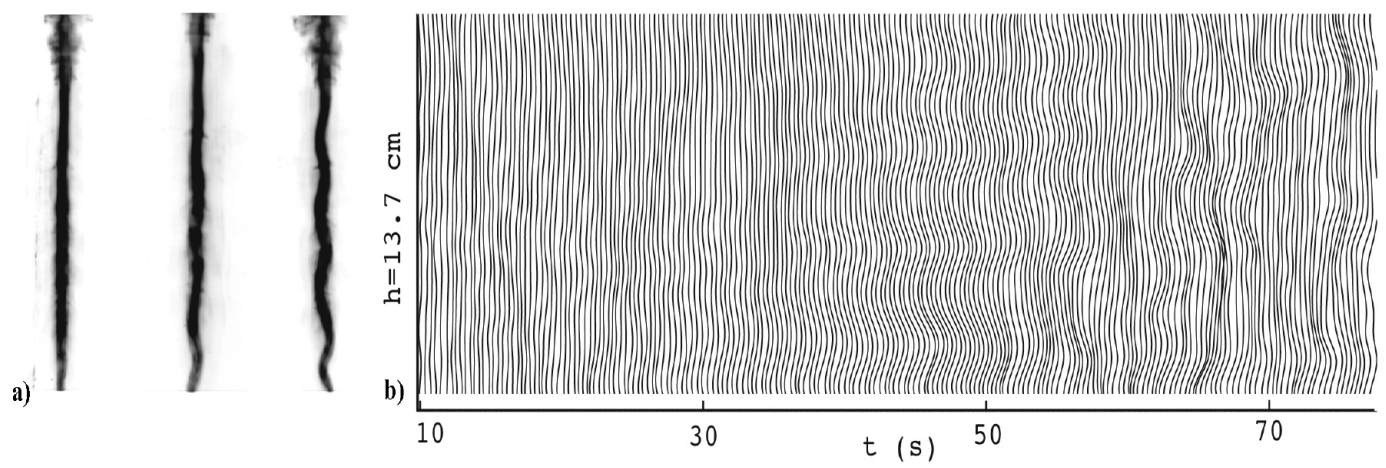

Fig. 4. (a) Side views of the vortex core at 5, 33 and $45 \mathrm{~s}$ after starting the flap motion visualized with fluorescent dye, and (b) the core centre-line as a function of time. The Froude number is 4.5 and the vertical size of the image is $17 \mathrm{~cm}$.

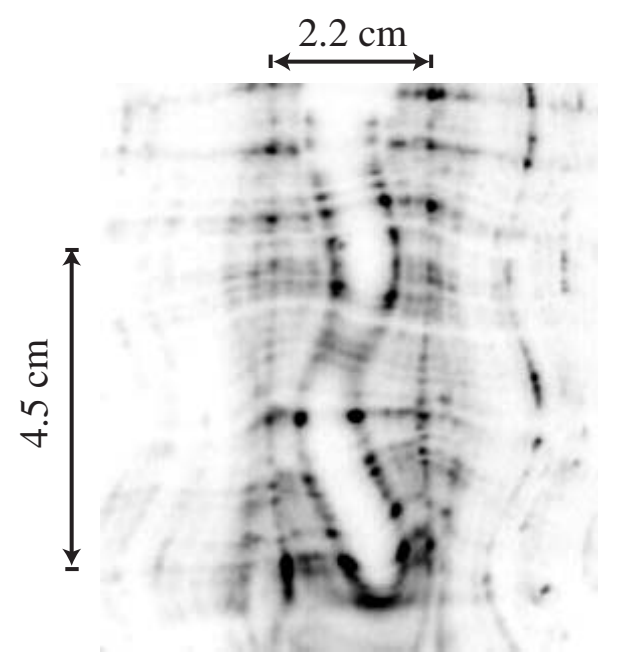

Fig. 5. Side view of the principal vortex cross section colored with dye at $t=38 \mathrm{~s}$ after its generation.

with $\Gamma_{1}$ and $\Gamma_{2}$ the circulation of the vortices and $b_{e q}$ their separation distance. If $\Gamma_{1} \neq \Gamma_{2}$ and the circulations are of opposite sign, the curvature of the trajectory of the most intense vortex, having circulation $\Gamma_{2}$, is

$b_{2}=\frac{\Gamma_{1} b_{e q}}{\Gamma_{1}+\Gamma_{2}}$.

With $\Gamma_{1}=14 \mathrm{~cm}^{2} / \mathrm{s}$ and $\Gamma_{2}=-70 \mathrm{~cm}^{2} / \mathrm{s}$ and a separation distance of $b_{e q}=10 \mathrm{~cm}$ this yields an angular velocity of $0.1 \mathrm{rad} / \mathrm{s}$, compared to $0.1 \pm 0.01 \mathrm{rad} / \mathrm{s}$ measured from experimental observations over half a period of rotation. Using these values we can derive the external strain on the vortices with

$S_{e 2,1}=\frac{\Gamma_{1,2}}{2 \pi b_{e q}^{2}}$,

implying an external strain of $0.02 \mathrm{~s}^{-1}$ for the principal vortex and $0.11 \mathrm{~s}^{-1}$ for the secondary vortex. Note that, due to

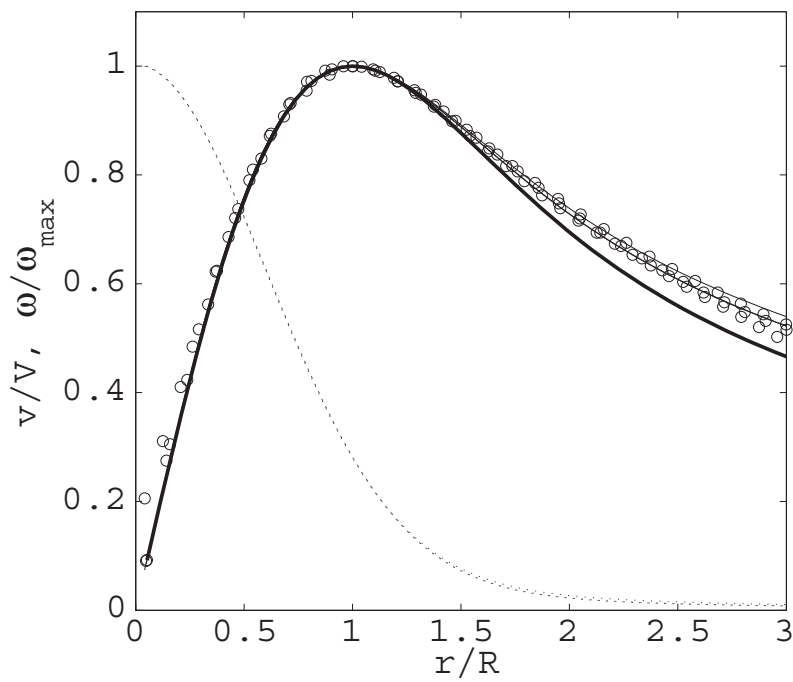

Fig. 6. Experimental velocity profiles over a vortex cross-section (circles) at $t=12 \mathrm{~s}$ after vortex generation for three different experiments, fitted with the vortex model Eq. (1) (solid curves). The thick curve correspond to the vortex model with a Gaussian distribution of vorticity. The dashed curve represents the vorticity profile corresponding to the fitted profiles.

the presence of vorticity in e.g. a Gaussian vortex, the strain increases towards the centre of the vortex. For the elliptical instability occurring in the vortex core, we thus need the value of the internal strain in the vortex centre. The relation between internal and external strain rate is given by $S_{i}=K S_{e}\left(R_{\omega}\right)$, with $R_{\omega}$ the second order vorticity moment

$R_{\omega}^{2}=\frac{1}{2 \Gamma} \int r^{2} \omega d S$

with $r$ the distance to the vortex centre. We estimate the radii of each vortex as the radius at which a Gaussian vorticity-distribution has a maximum azimuthal velocity, i.e. $R=1.12 R_{\omega}$. During the early stages of the dipole evolution, the measurements yield $2.5 \mathrm{~cm}$ and $1.3 \mathrm{~cm}$ for the 


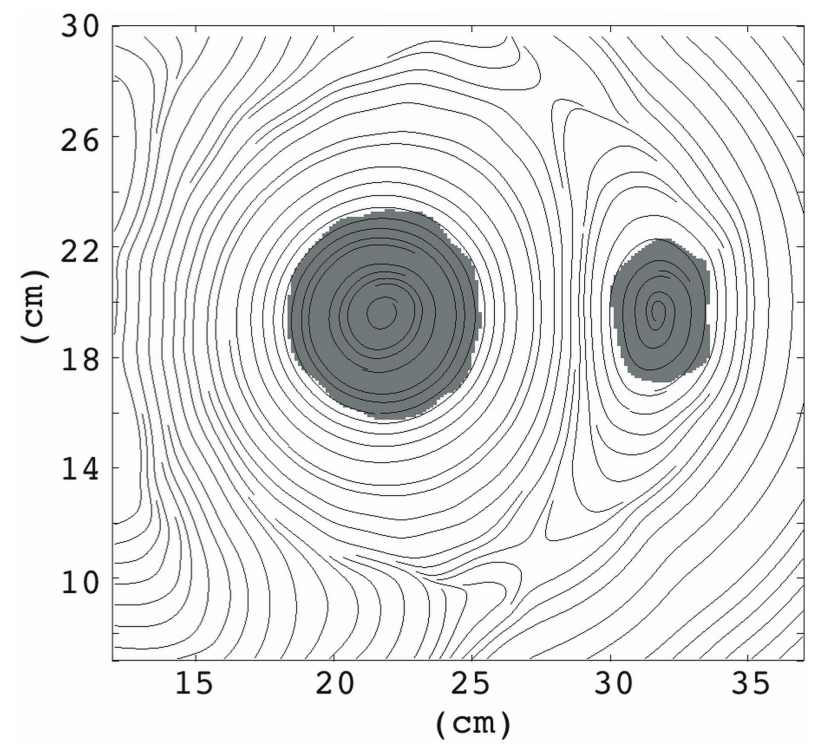

Fig. 7. Stream lines measured in the reference frame moving with the dipole structure. Gray areas represent vorticity exceeding $10 \%$ of the maximum vorticity.

principal and secondary vortex, respectively, whereas after $30 \mathrm{~s}$, these radii are $2.7 \mathrm{~cm}$ and $1.7 \mathrm{~cm}$. With these values the value of $K$ is given by (see le Dizès and Laporte, 2002)

$K(\mu)=1.5+0.038\left(0.16-\frac{\Omega_{p}}{2 \mu}\right)^{-9 / 5}$,

where $\mu=\Gamma /\left(2 \pi R_{\omega}^{2}\right)$ is the maximum angular velocity and $\Gamma$ the circulation of the vortex. This gives a factor $K$ close to 3 for the principal vortex whether the radius is $2.5 \mathrm{~cm}$ or $2.7 \mathrm{~cm}$. For the secondary vortex, this factor varies from 3.2 to 4.1 for a radius of $1.3 \mathrm{~cm}$ or $1.7 \mathrm{~cm}$, respectively. The growth rate of an inertial wave with inclination $\xi$ and vertical wave number $k_{z}$ is:

$$
\begin{aligned}
\sigma & =\sqrt{\left(\frac{3 \mu-\Omega_{p}}{4 \mu}\right)^{4} S_{i}^{2}-\left(\mu-\Omega_{p}-2 \mu \cos \xi\right)^{2}} \\
& -v \frac{k_{z}^{2}}{\cos ^{2} \xi},
\end{aligned}
$$

where $v$ is the viscosity of the fluid. In this expression, the last rhs-term takes into account the viscous damping of the perturbation. The relation between the angle of the inertial wave to the vertical wavelength is given by

$\cos \xi=\frac{1}{2}-\frac{(2.26+1.69 n)-k_{z} R_{\omega}}{14.8+9 n}$,

for an inertial wave of azimuthal wave-number 1 , with $n=0,1,2 \ldots$ the index of the branch of the dispersion relationship. The maximum growth rate for the elliptical instability of the principal vortex yields $0.010 \mathrm{~s}^{-1}$ for a radius of $2.5 \mathrm{~cm}$ and $0.015 \mathrm{~s}^{-1}$ for $2.7 \mathrm{~cm}$. The maximum growth rate of the weakest secondary vortex becomes $0.045 \mathrm{~s}^{-1}$ for a radius of $1.7 \mathrm{~cm}$. The typical wavelength obtained with the largest radii is $3.5 R_{\omega}$ for the starting vortex and $1.6 R_{\omega}$ for the stopping vortex. This wavelength is slightly outside the validity domain of the asymptotic approach of le Dizès and Laporte (2002). The presence of a stable density stratification modifies these values (see Miyazaki and Fukumoto, 1992).

The expression of the growth rate given by Kerswell (2002) for a stratified fluid in solid body rotation as basic flow is:

$\sigma^{*}=\frac{9}{16} \epsilon^{*} \frac{4\left(3+2 \Omega_{p}^{*}\right)^{2}\left(1-N^{* 2}\right)}{94\left(1+\Omega_{p}^{* 2}\right)-N^{* 2}}$

where $\epsilon^{*}$ is the non-dimensional strain-rate. Variables are normalized by the angular velocity of the flow. For a homogeneous fluid, the growth rate can be estimated by the expression for internal strain of a purely elliptical flow $\epsilon=S_{i}$ (Eloy and le Dizès, 1999). Since the basic flow is two dimensionnal, this is also true for a stratified fluid. This theoretical growth rate is represented as a function of Froude number in Fig. 8, and compared to the growth rate in a homogenous fluid according to le Dizès and Laporte (2002) including viscous damping. Note that here, the Froude number is defined as $F r=\Omega / N$ which equals $U / N R$ in case of a rotating cylinder or Rankine vortex. For lower Froude number values the growth rate decreases, whereas it reaches a constant value for higher Fr values. The homogeneous limit of le Dizès and Laporte (2002) with (and without) viscous damping of the unstable mode is also reported. Since the instability of the presently studied vortices is heavily affected by the nonlinear vortex interaction, we use these values as an order of magnitude.

\subsection{The instability wave-length of the principal vortex}

The wavelength of the instability is measured simultaneously with maximum velocity and radius of the vortex, using measurements with a top and side-view camera, and is displayed against Froude number in Fig. 10. With increasing Froude number the wavelength decreases until reaching a constant value that is predicted by the theory for a Oseen-like vortex subject to a weak external strain in a homogeneous fluid (see Eloy and le Dizès, 1999). This theoretical value for the wavelength (dashed line in Fig. 10) corresponds to the resonance condition of two inertial modes of azimuthal wave number 1 and a pulsation equal to zero. The choice of zero pulsation is justified by the fact that the growth rate of the instability is stationary in all experiments (see Fig. 4). Also the structure of the radial perturbation (see Fig. 5) defines the unstable wave length found by Eloy and le Dizès (1999) for a Gaussian vortex in homogeneous fluid. To compare this wave length with our results, we scale with the radius of maximum velocity which gives $\lambda / R=2.5$. 
The solid curve of the Fig. 10 is obtained from a simplified model used to verify the dependence of the instability wavelength on Froude number. The basic mechanism of the elliptical instability involves a resonant interaction between Kelvin (or inertial) waves. These waves were first identified on a cylindrical column of fluid in solid-body rotation by Kelvin (1880) and are described by harmonic functions of the kind:

$x^{\prime}=x(r) e^{i(l \theta+m z-\omega t)}$,

where $x^{\prime}$ stands for any component of the perturbed field, $l$ the azimuthal wavenumber, $m$ the axial wavenumber and $\omega$ is the wave pulsation. When a stationary ellipticity is imposed by an external horizontal strain field of mode 2 , Kelvin helical waves $(|l|=1)$ with the same wavenumber and zero pulsation resonate (see e.g. Kerswell, 2002), leading to the so-called elliptical instability. The same mechanism still holds for a Gaussian vortex, although the vorticity gradient strongly modifies the dispersion relationship (see e.g. Eloy and le Dizès, 1999).

For a density stratification with a buoyancy frequency, $N$, lower than the maximum of vorticity, these inertial waves still exist but the dispersion relationship is modified (see Miyazaki and Fukumoto, 1991).

Kerswell (1992) considered the elliptical instability of waves in a fluid in solid body rotation with axial stratification and derived the dispersion relationship for both cases, with and without magnetic field. Here, we consider the simplest case. Solid body rotation is chosen for its simplicity, its relevant physics and as a complementary diagnostic tool, with in the limit, the wavelength for a Gaussian vortex in a homogeneous fluid. The difference in the velocity profile, however, affects the wavelength. The purpose of this model is to compare qualitatively the variation in wave-length with Froude number with the variation in our experimental data.

From a superposition of an axisymmetric two-dimensional flow and the small harmonic perturbation we obtain the equation for the pressure perturbations. The basic flow field $U=(0, V(r), 0)$ and perturbed field $U+u^{\prime}=\left(u^{\prime}, V+v^{\prime}, w^{\prime}\right)$ are supposed to satisfy the Euler equations in Boussinesq approximation. After linearization, the perturbation equations for momentum, density and continuity become, respectively,

$\left(\Phi-\sigma^{2}\right) u=i \sigma \frac{d \tilde{p}}{d r}-i \frac{2 l \Omega}{r} \tilde{p}$,

$\left(\Phi-\sigma^{2}\right) v=Z \frac{d \tilde{p}}{d r}-\frac{l}{r} \sigma \tilde{p}$,

$\left(N^{2}-\sigma^{2}\right) w=-m \sigma \tilde{p}$,

$\frac{1}{r} \frac{d}{d r}(r u)+\frac{i l}{r} v+i m w=0$.

where $\tilde{p}=p / \rho_{0}$ with $p$ the amplitude of the pressure perturbation, $\Omega=V / r, Z=1 / r(d / d r)(r V), \sigma=\omega-l \Omega$ is the doppler shifted frequency and $\Phi=2 \Omega Z$ is the Rayleigh discriminant. After some manipulation of the set (10d), one

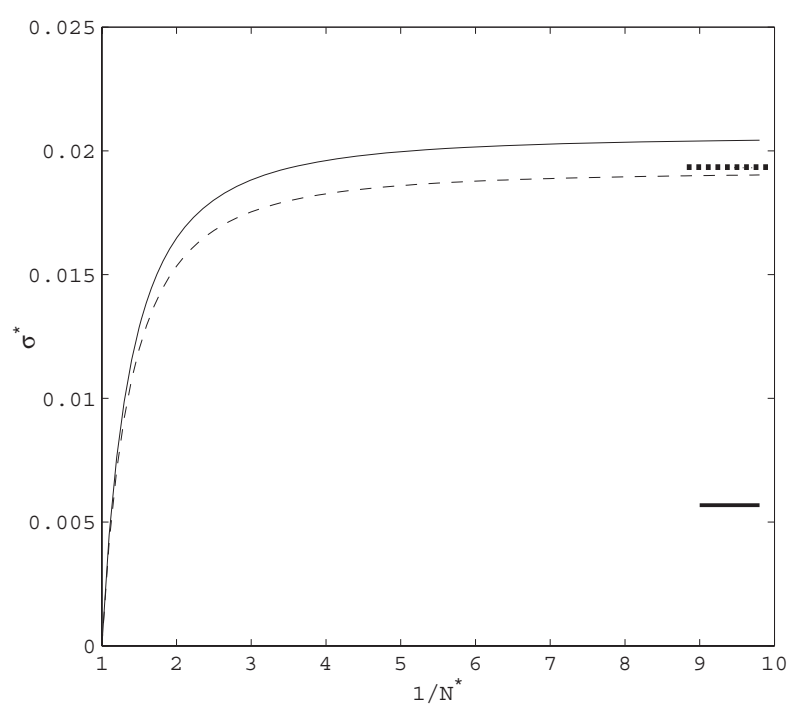

Fig. 8. Theoretical growth rate of the elliptical instability as a function of the Froude number. The continuous curve is the rotating stratified case, the long dashed curve is the non rotating case, both from Kerswell (2002). The small dashed and thick lines represent, respectively, the non-viscous and viscous homogeneous limit from le Dizès and Laporte (2002).

obtains the Bessel equation

$r^{2} \frac{d^{2} p}{d r^{2}}+r \frac{d p}{d r}+\left(\frac{\Phi-\sigma^{2}}{\sigma^{2}-N^{2}} m^{2} r^{2}-l^{2}\right) p=0$.

As boundary condition we take a non-singular condition at the centre $r=0$, and with (10d)a, a zero radial velocity condition at some radius $R$. The choice of a bounded flow yields a wave number selection, in contrast to an infinite flow. Regularity at $r=0$ excludes Bessel functions of the second kind. The second condition yields the dispersion relationship (see e.g. Chandrasekhar, 1981):

$\sigma^{*}= \pm \sqrt{\frac{4+\left(N^{*} \alpha / m^{*}\right)^{2}}{1+\left(\alpha / m^{*}\right)^{2}}}$,

where $N^{*}=N / \Omega, m^{*}=m R$ and $\sigma^{*}=\sigma / \Omega$. The parameter $\alpha$ is the solution of

$\alpha J_{l}^{\prime}(\alpha)+\frac{2 l \Omega}{\sigma} J_{l}(\alpha)=0$,

with $J_{n}(x)$ the Bessel function of order $n$. Equation (12) shows that $N<\sigma<2 \Omega$. Moreover, the effect of stratification is negligible for small $N^{*}$ and large $m^{*}$, which is verified for $F r>2$ (i.e. $N^{* 2}<1 / 4$ ) and for the short wavelength elliptical instability. The secant method is used to find the roots of Eq. (13) and the same method is used to find the roots of the dispersion relationship for the azimuthal mode $l=1$ qiven by Eq. (12). Figure 10 represents the variation of the second root. The three first roots show the same tendency. We have taken the second since it is the closest to the data. 


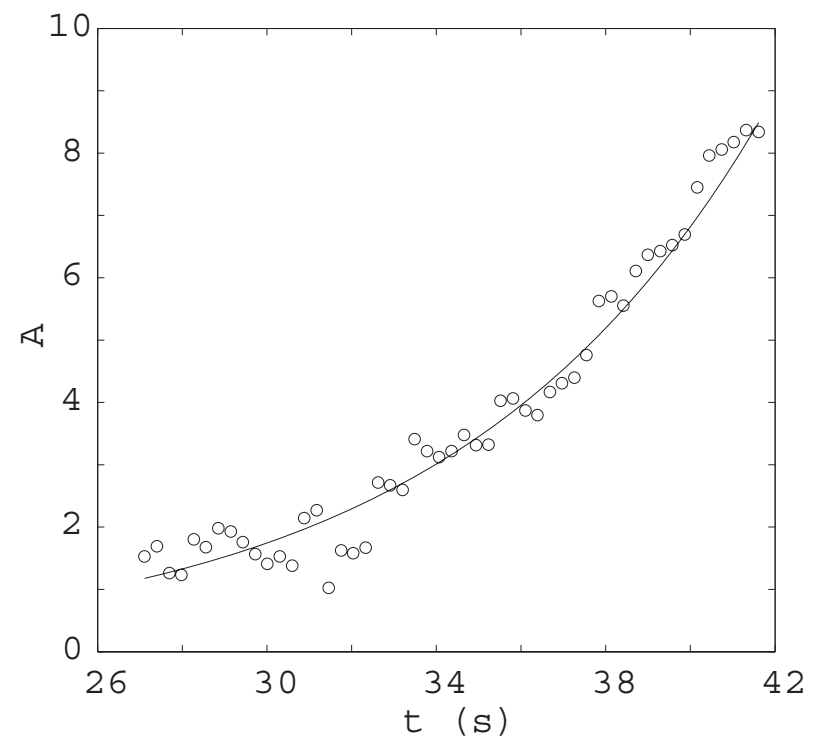

Fig. 9. Measurement of the amplitude of the instability of the vortex core (dots, arbitrary unit) as a function of time. The solid curve is the exponential fit which gives a growth rate of $0.13 \mathrm{~s}^{-1}$.

\subsection{The growth rate of the principal vortex}

The growth rate of the instability was measured from dye visualizations of the vortex core, which continuously deforms until the amplitude saturates (see Fig. 4). For these Froudenumber values, stratification effects are relatively weak (see Fig. 10). The increase in amplitude is measured by fitting the data with an exponential function (see Fig. 9) yielding a growth rate of $0.13 \mathrm{~s}^{-1}$ for $F r \approx 4$. This growth rate is about 10 times larger than predicted by the theory based on the initial ellipticity of the primary vortex. As shown in the sequence of images in Fig. 2, the nonlinear interaction with the unstable secondary vortex significantly increases this ellipticity. In order to estimate the strain on this primary vortex we suppose it is a dipolar vortex. Then, with the theory of le Dizès and Laporte (2002) for a symmetric dipolar vortex we determine the distance between the two vortices, $b$, yielding a measure for the corresponding strain. This allows us to normalize the growth rate with the external strain $S_{e}=\Gamma /\left(2 \pi b^{2}\right)$ and compare the scaled growth rate, $\sigma^{*}=\sigma / S_{e}$, with that of mutually strained vortices in a symmetric dipole Leweke and Williamson (1998). In a symmetric dipole the ellipticity of the vortices and the strain induced on the "principal vortex" are much larger than for the initially asymmetric dipole. For a vortex radius $2.5 \mathrm{~cm}$ and circulation $70 \mathrm{~cm}^{2} \mathrm{~s}^{-1}$, we obtain $\sigma^{*}=1.2$. For an equivalent ratio $R / b$ of a symmetric dipole (Leweke and Williamson, 1998, see) find a growth rate equal to 0.94 . The difference may be explained by the difference in circulation-based Reynolds number which was of the order 7000 compared to 2800 in the experiments of Leweke and Williamson (1998). When

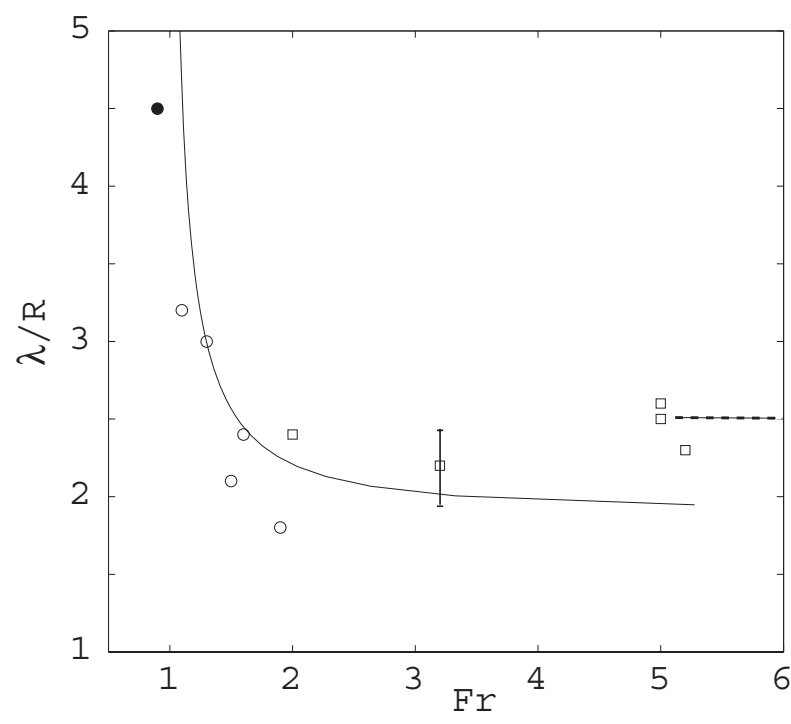

Fig. 10. Measurements of the core instability wavelength as a function of the Froude number. Squares correspond to the $80 \times 80 \mathrm{~cm}^{2}$ tank, circles to the $40 \times 40 \mathrm{~cm}^{2}$ tank and the filled circle corresponds to the instability of the secondary vortex. The curve corresponds to the variation obtained from Eq. (12) and the dashed line represents the homogeneous limit.

neglecting viscous effects the theory of le Dizès and Laporte (2002) yields an even higher value for a symmetric dipole, $\sigma^{*}=1.42$. This shows that the growth rates for $F r>4$ correspond reasonably well to the growth rate found for a symmetric dipolar vortex in a homogeneous fluid. This growth rate is also close to the growth rate, $\sigma^{*}=1.32$, obtained by Miyazaki and Fukumoto (1992) for an elliptical vortex in a stratified fluid.

\section{Conclusions}

We have explored the elliptical instability of highly asymmetric vortex pairs in a weakly stratified fluid for Froude numbers $F r \leq 1$. Our results correspond to the numerical results of Billant and Chomaz (2000b); the wavelength of the instability equally increases from $\lambda / R \approx 2.5$ for $\mathrm{Fr}=5$ to $\lambda / R \approx 4$ for $F r=1$. For $F r>2$, (see Fig. 10) we show that the properties of the elliptical instability are quantitatively close to theoretical and numerical results obtained for a homogeneous fluid by le Dizès and Laporte (2002). For decreasing Froude number the wavelength increases between $1<F r<2$, whereas the instability is suppressed for Froude numbers $F r<1$. This variation is in good agreement with the theory for the simplified case of a bounded cylindrical body of stratified fluid in solid rotation.

An interesting result of the present investigation is that the nonlinear interaction of the secondary vortex with the principal vortex significantly increases the ellipticity of the 
principal vortex. The instability growth rate increases by a factor ten. This, even though the secondary vortex has a 10 times weaker circulation and could be considered of minor importance. This suggests that the elliptical instability of the core of the principal vortex may also be be relevant in stratified fluids for Froude numbers $\geq 1$.

Acknowledgements. The authors greatly acknowledge the technical assistance of P. Carecchio. J.-B. Flór acknowledges S. Dalziel for the use of DigImage during his visit at the DAMTP laboratory between 1994-1996. The work was funded by the program of CAT-NAT "Genèse et instabilités des fronts et cyclones intenses" of the CNRS.

Edited by: W.-G. Früh

Reviewed by: three referees

\section{References}

Afanasyev, Y. D.: Experminents on instability of columnar vortex pairs in rotating fluid, Geophys. Astrophys. Fluid Dyn., 96(1), 31-48, 2002.

Billant, P. and Chomaz, J.-M.: Experimental evidence for a new instability of a vertical columnar vortex pair in a strongly stratified fluid, J. Fluid Mech., 418, 167-188, 2000a.

Billant, P. and Chomaz, J.-M.: Three-dimensional stability of a vertical columnar vortex pair in a stratified fluid, J. Fluid Mech., 416, 65-91, 2000b.

Chandrasekhar, S.: Hydrodynamic and hydromagnetic stability, DOVER PUBLICATIONS, INC., 1981.

Dalziel, S. B.: Decay of rotating turbulence: some particle tracking experiments, in: Flow visualization and image analysis, edited by: Nieuwstadt, F. T. M., pp. 27-54, Kluwer, Dordrecht, 1993.

Dalziel, S. B., Hughes, G. O., and Sutherland, B. R.: Whole-field density measurement by 'synthetic schlieren', Exps. fluids, 28, 322-335, 2000.
Eloy, C. and le Dizès, S.: Three-dimensional instability of Burgers and Lamb-Oseen vortices in a strain field, J. Fluid Mech., 378, 145-166, 1999.

Fincham, A. M. and Delerce, G.: Advanced optimization of correlation imaging velocimetry algorithms, Exps. fluids, Suppl., S13S22, 2000.

Fincham, A. M. and Spedding, G. R.: Low cost, high resolution DPIV for measurement of turbulent fluid flow, Exps. fluids, 23, 449-462, 1997.

Flór, J.-B. and Eames, I. E.: The dynamics of monopolar vortices on the $\beta$-plane, J. Fluid Mech., 456, 353-376, 2002.

Honyi, H., Taneda, S., and Tatsudo, M.: Some practical details of the electrolytic precipitation method of flow visualization, Rep. Res. Inst. Appl. Mech., 28, 83-89, 1980.

Kelvin, L.: Vibration of a columnar vortex, Phil. Mag., 10, 155$168,1880$.

Kerswell, R. R.: Elliptical instabilites of stratified, hydromagnetic waves, Geophys. Astrophys. Fluid Dyn., 71, 105-143, 1992.

Kerswell, R. R.: Tidal excitation of hydromagnetic waves and their damping in the Earth, J. Fluid Mech., 274, 219-241, 1994.

Kerswell, R. R.: Elliptical instability, Annu. Rev. Fluid Mech., 34, 83-113, 2002.

Kloosterziel, R. C.: The vortices of two-dimensional turbulence, $\mathrm{Ph} . \mathrm{D}$. thesis, University of Utrecht, 1990.

le Dizès, S. and Laporte, F.: Theoretical predictions for the elliptical instability in a two-vortex flow, J. Fluid Mech., 471, 169-201, 2002.

Leweke, T. and Williamson, C. H. K.: Cooperative elliptic instability of a vortex pair, J. Fluid Mech., 360, 85-119, 1998.

Miyazaki, T. and Fukumoto, Y.: Axisymmetric waves on a vertical vortex in a stratified fluid, Phys. Fluids, A3(4), 606-616, 1991.

Miyazaki, T. and Fukumoto, Y.: Three-dimensional instability of strained vortices in a stably stratified fluid, Phys. Fluids, A 4(11), 2515-2522, 1992.

Oster, G.: Density gradient, Sci. Am., 212, 70-76, 1965.

Thomas, P. J. and Auerbach, D.: The observation of the simultaneous development of a long- and short-wave instability mode on a vortex pair, J. Fluid Mech., 265, 289-302, 1994. 